\title{
Clinical outcome of septic patients with undetectable vitamin D levels at ICU admission
}

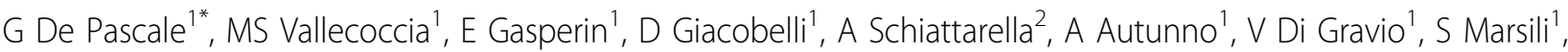 \\ SL Cutuli ${ }^{1}$, MA Pennisi ${ }^{1}$, C Zuppi $^{2}$, SA Quraishi ${ }^{3}$, M Antonelli ${ }^{1}$ \\ From ESICM LIVES 2015 \\ Berlin, Germany. 3-7 October 2015
}

\section{Introduction}

Septic patients with very low vitamin D (VD) levels are expected to most benefit from supplementation strategies but few data are available in this specific population [1].

\section{Objectives}

Our purpose is to investigate the clinical/epidemiological profile and sepsis-related outcome of critically ill septic patients with undetectable VD levels at ICU admission.

\section{Methods}

We conducted an observational study enrolling, during a 12 months period, consecutive patients admitted to our ICU with severe sepsis/septic shock.

\section{Results}

170 blood samples were obtained from 107 patients (septic shock / severe sepsis: 62\% / 38\%). ICU admission VD deficiency $(\leq 20 \mathrm{ng} / \mathrm{mL})$ was observed in $93.5 \%$ of the patients: 57 (53.3\%) showed undetectable levels $(<7 \mathrm{ng} / \mathrm{mL})$. In patients $(\mathrm{n}=33)$ who received, during the ICU stay, more than one VD blood sampling, hypovitaminosis $\mathrm{D}$ category did not change over time $(\mathrm{p}=\mathrm{ns})$. The principal infection site was the lung (48.6\%): $50(46.7 \%)$ patients were bacteraemic. Comparing patients with undetectable VD levels with those ones with values $\geq 7 \mathrm{ng} / \mathrm{mL}$, there were not significant differences regarding main comorbidities, presenting features and disease severity $(p=\mathrm{ns})$. The former group showed a higher rate of microbiologically confirmed infections but a lower percentage of microbiological eradication $(80.7 \%$ vs. $58 \%, p=0.02$; $35.3 \%$ vs $68 \% ; p=0.03$, respectively).
Furthermore they experienced longer duration of mechanical ventilation and vasopressor support: $9 d s$ [3.75-12.5] vs. $4 d s$ [2-0], $p=0.04 ; 7 d s$ [4-10] vs. $4 d s$ [2-7.25], $p=0.02$. Sepsis-related mortality rate was higher in patients with VD levels $<7 \mathrm{ng} / \mathrm{mL}(50.9 \%$ vs $26 \%$ ). Multivariable regression analysis confirmed ICU admission undetectable VD concentration $(\mathrm{p}=0.01)$ as independent predictor of sepsis-related mortality.

\section{Conclusions}

Our results suggest that in critically ill septic patients undetectable VD levels at ICU admission may be a major determinant of clinical outcome. Further studies should assess the impact of replacement strategies in this subgroup of patients.

\section{Authors' details}

'Sacro Cuore Catholic University, A. Gemelli Hospital, Department of Anesthesiology and Intensive Care, Rome, Italy. ${ }^{2}$ Sacro Cuore Catholic University, A. Gemelli Hospital, Institute of Biochemestry and Clinical Biochemestry, Rome, Italy. ${ }^{3}$ Harvard Medical School, Massachusetts General Hospital, Department of Anesthesia, Critical Care and Pain Medicine, Boston, United States.

Published: 1 October 2015

\section{Reference}

1. Amrein $\mathrm{K}$, et al: Effect of high-dose vitamin D3 on hospital length of stay in critically ill patients with vitamin D deficiency: the VITdAL-ICU randomized clinical trial. Jama 2014, 312(15):1520-30.

\section{doi:10.1186/2197-425X-3-S1-A80}

Cite this article as: De Pascale et al:: Clinical outcome of septic patients with undetectable vitamin D levels at ICU admission. Intensive Care Medicine Experimental 2015 3(Suppl 1):A80. 